\title{
Chondrogenesis induced from human embryonic stem cells
}

The potential use of human embryonic stem cells (ESCs) in regenerative medicine is unquestionable, but in the context of cartilage engineering, efforts to differentiate human ESCs into articular chondrocytes have met with little success. Reporting in Arthritis \& Rheumatism, Nakagawa and colleagues describe how bone morphogenetic protein 7 (BMP7) and transforming growth factor $\beta 1$ (TGF- $\beta 1$ ) can induce chondrogenesis in human ESCs.

Directly plating human ESCs onto tissue-culture-treated plastic plates has uncovered osteogenic and adipogenic potentials for these cells, so Nakagawa et al. investigated the chondrogenic potential of human ESCs derived by direct plating followed by three-dimensional pellet cultures. Importantly, this method avoids the formation of embryoid bodies, which often results in heterogeneous chondrogenic differentiation. The pellets, generated by centrifuging the cells at $200 \mathrm{~g}$ for $5 \mathrm{~min}$, were cultured in chondrogenic medium alone, or chondrogenic medium containing TGF- $\beta 1$, or BMP7, or TGF- $\beta 1$ plus BMP7. Addition of TGF- $\beta 1$ plus BMP7 to the chondrogenic medium resulted in the largest increase in size and weight of the pellets after 14 days of in vitro chondrogenesis, which the researchers attributed to an increase in the biosynthesis of sulfated glycosaminoglycan (sGAG) accompanied by the accumulation of water: treatment with chondrogenic medium and TGF- $\beta 1$ plus BMP7, or chondrogenic medium plus $\mathrm{BMP} 7$ resulted in a larger increase in the content of sGAG in the pellets than treatment with chondrogenic medium alone or with the addition of only TGF- $\beta 1$.

In pellets cultured in chondrogenic medium plus BMP7, only the periphery showed well-differentiated chondrocytes and stained strongly for GAG and type II collagen; strong staining for GAG and abundant differentiated mature chondrocytes were seen in pellets cultured in chondrogenic medium plus
BMP7 and TGF- $\beta 1$. When they analyzed the expression levels of aggrecan and type II collagen II, the researchers noted that BMP7 strongly induced these cartilage-specific genes.

Although Nakagawa et al. have successfully induced chondrogenesis in human ESCs using a reproducible method, superficial zone protein (also known as lubricin) could not be detected in the pellet cultures, implying that chondrocytes specific to the superficial zone of articular cartridge were absent. The researchers are, however, hopeful that optimization of the conditions for in vitro chondrogenesis might permit the expression of the articular cartilage phenotype.

\section{Katrin Legg}

Original article Nakagawa, T. et al. Induction of chondrogenesis from human embryonic stem cells without embryoid body formation by bone morphogenetic protein 7 and transforming growth factor $\beta 1$. Arthritis Rheum. 60, 3686-3692 (2009) 